\title{
L'ÉCRÊTEMENT DES SINISTRES “AUTOMOBILE”
}

\author{
Par Lionel Moreau \\ Assurances Générales de France, Paris
}

\begin{abstract}
SUMMARY
French insurance companies usually classify their agents according to their results by branch and to this effect they calculate their respective claim ratios.

As regards motor insurance many agents have argued against this practice as they believed it was unfair to be adversely classified when they had the misfortune to incur a large claim.

In this article, the merits of various statistical systems for attenuating the effects of large claims on the portfolio are considered in order to come to the most equitable solution possible, i.e., to ascertain the best method and system for attenuating the effects of large claims. The conclusion is that the excess of loss technique is best suited to this effect.

To arrive at this conclusion the author of this article relates in detail the various stages of the study, the various systems envisaged and the tests which have allowed to select the most appropriate system. The main results obtained are also given together with their respective drawbacks.

At the end of the article the author mentions the still fairly limited bibliography which deals with this question.

Dans le but d'améliorer les résultats, les compagnies d'assurance ont l'habitude en France de publier un classement de leurs Agents Généraux basé sur le ratio $S / P$ (sinistres/primes) et de tenir compte de ce classement pour l'attribution de certains avantages commerciaux.

Or, en assurance automobile, un malaise profond s'était emparé d'un certain nombre d'entre eux, et ils avaient mis l'accent sur un point qu'ils estimaient injuste:

"Nous ne pouvons pas être pénalisés" disaient-ils, "si un très gros sinistre, atteignant 500 ou 1000 fois la prime annuelle, frappe un de nos clients, car il s'agit alors d'un phénomène aléatoire dont nous ne sommes pas responsables".

Telle est l'origine de la politique de l'écrêtement.
\end{abstract}

\section{LA THEORIE DE BASE}

Nous désignerons pour une agence $i$ :

- les primes émises par $P_{i}$

- le nombre de véhicules par $v_{i}$

- le nombre de sinistres par $n_{i}$

- le montant des sinistres par $S_{i}$

- le montant du sinistre $j$ par $s_{j}^{j}$

$$
S_{i}=\sum_{j=1}^{n_{i}} s_{i}^{j}
$$

ASTIN BULLETIN Vol. 14, No. 2 
Nous voulons substituer à l'indicateur $S_{i} / P_{i}$ brut un indicateur plus nuancé, obtenu par la répartition sur l'ensemble des agences de la masse des montants des sinistres excédant un seuil nommé priorité. Cette technique est celle bien connue en réassurance sous le nom d'excess-loss. Pour utiliser le langage habituel, nous parlerons des sous-crêtes, pour désigner les montants inférieurs à cette priorité et surcrêtes les montants supérieurs.

On peut donc se poser deux problèmes distincts:

(a) Comment calculer les surcrêtes?

(b) Comment les répartir?

(a) pour les calculer, trois méthodes sont proposées par Mlle Hess (cf. bibliographie [1]):

1. La priorité fixe

Doit alors être répartie, pour l'ensemble des agences, la masse $R$ des sinistres supérieure à la priorité fixe $F$ :

$$
R=\sum_{i} \sum_{j \in J_{i}}\left(S_{i}^{j}-F\right)
$$

avec $J_{i}=\left\{j: s_{i}^{j}>F\right\}$.

2. La priorité dite au Kème percentile

On veut par exemple écrêter $1 \%$ ou $2 \%$ des sinistres. La priorité $F$ est alors le résultat d'un calcul implicite:

$$
\sum_{i} \sum_{j \in J_{i}}=K \cdot \sum_{i} n_{i}
$$

et on détermine aisément $F$ par le tracé de l'histogramme cumulatif.

3. La priorité dite au percentile variable

C'est la même technique mais segmentée, les agences ayant été préalablement ventilées dans des classes différentes en fonction de critères de taille, le pourcentage pouvant devenir plus important si la taille est plus petite, afin de minimiser la dispersion.

(b) Pour répartir cette masse, on peut appliquer plusieurs techniques:

1. Répartition sur les sous-crêtes:

Si nous posons

$$
\begin{aligned}
& T_{i}=\sum_{j \notin J_{i}} s_{i}^{j}+F \sum_{j \in J_{i}} 1 \\
& S_{i}^{\prime}=T_{i} \times\left(1+\frac{R}{\sum_{i} T_{i}}\right) .
\end{aligned}
$$

2. Répartition sur les nombres de sinistres:

$$
S_{i}^{\prime}=T_{i}+\frac{R \times n_{i}}{\sum_{i} n_{i}}
$$


3. Répartition sur les nombres de véhicules:

$$
S_{i}^{\prime}=T_{i}+\frac{R \times v_{i}}{\sum_{i} v_{i}} .
$$

4. Nous ajouterons bien sûr une quatrième répartition, la répartition sur les primes

$$
S_{i}^{\prime}=T_{i}+\frac{R \times P_{i}}{\sum P_{i}}
$$

On doit donc étudier quel indicateur rendra le mieux compte de la situation.

Les travaux mentionnés ci-dessus nous proposent de déterminer $S_{i}^{\prime} / P_{i}$ ayant l'une des formes 1,2 ou 3 ci-dessus.

Nous proposons d'y ajouter la quatrième et même d'étudier une forme plus classique, celle appliquée en réassurance d'excess-loss, à savoir:

$$
\begin{gathered}
T_{i} / P_{i}^{\prime} \\
P_{i}^{\prime}=P_{i} \times\left(1-\frac{R}{\sum P_{i}}\right)
\end{gathered}
$$

qui consiste à ne prendre en compte que les sous-crêtes rapportées à des primes nettes du prix de l'excess.

\section{CRITERE D'OPTIMISATION}

Entre toutes ces méthodes d'écrêtement et ces choix de répartition et d'indicateur, quelle est la meilleure façon de procéder? Celle bien sûr qui donne le meilleur ajustement avec ce que nous déciderons d'appeler le meilleur critère (ce qui résulte là d'un choix et non d'une vérité qui s'imposerait).

Nous avons pensé que les agents étaient "responsables" des éléments qualitatifs suivants:

- les sinistres matériels en fréquence et en coût moyen

- les sinistres corporels en fréquence.

Autrement dit, nous voulions juger les agents sur le critère $\hat{S}_{i} / P_{i}$

avec:

$$
\hat{S}_{i}=\sum_{j \in J_{i}^{m}} s_{i}^{j}+m \sum_{j \in J_{i}^{c}} 1
$$

$$
J_{i}^{m}=j: s_{i}^{j}=\text { montant d'un sinistre matériel }
$$

et

$$
J_{i}^{c}=j: S_{i}^{\prime}=\text { montant d'un sinistre corporel }
$$

$m$ étant le coût moyen national:

$$
m=\left(\sum_{i} \sum_{j \in J_{i}^{c}} s_{i}^{j}\right) / \sum \sum_{i \in J_{i}^{c}} 1 .
$$


Nous ne prétendrons pas ici que ce critère était le meilleur, mais il était celui qui correspondait le mieux à la sensibilité de notre Compagnie et de l'Assurance Automobile française qui, par la pratique du bonus, a éliminé le coût moyen des gros sinistres comme critère de jugement, polarisant son action contre les excès de fréquence. Eliminer le coût réel des matériels ne nous a pas par contre semblé judicieux pour deux raisons:

(a) cela aurait introduit un trop gros biais avec une formule d'écrêtement

(b) les agents, par l'exercice rapide des recours, et une bonne connaissance du marché local peuvent avoir une influence, légère mais réelle, sur les coûts moyens des petits matériels.

\section{LE TRAVAIL ENTREPRIS}

Les pages qui vont suivre vont décrire l'étude à laquelle nous nous sommes livrés en 1979 pour le compte de notre Direction Automobile.

Nous cherchions au départ, en partant du support réel de nos agences, à déterminer quelle formule et quel niveau d'écrêtement se rapprochaient le plus du critère optimal que nous avons décrit plus haut.

Ce critère en effet, ne pouvait rester que théorique: Les esprits n'auraient pas été prêts à l'accueillir, et sur le plan pratique, il était très difficile à réaliser à l'état permanent. Nous étions tenus par deux contraintes:

1. les données dont nous disposions,

2. notre marge de négociation.

Le point 2 nous imposait déjà une solution d'écrêtement parce qu'elle avait déjà fait son chemin dans les esprits et nous recommandait la voie la plus simple, si possible une priorité fixe et une répartition en primes. Le point 1 nous obligeait à faire l'inventaire de nos données statistiques.

\section{A. Inventaire Statistique}

La branche Automobile dispose d'un grand nombre de ventilations statistiques: elle est d'ailleurs tenue par la réglementation de fournir des états faisant ressortir, pour de très nombreuses catégories, les ratios $S / P$ (Sinistres/Primes acquises) recalculés à chaque clôture d'exercice pour tous les exercices antérieurs (le taux n'étant absolument définitif que vers la 6ème année mais déjà très stabilisé après la 3ème). On peut donc distinguer les résultats des différentes garanties (responsabilité civile, dommages) dans les différents types de véhicules ( 2 roues, 4 roues, camions, transports publics, etc. ...) et même dans les différents types de garantie (contrats n'ayant que la Responsabilité Civile, ayant les dommages, etc. ...).

Malheureusement, et cela se comprend aisément, étant donné le nombre de divisions que cela créerait, ces statistiques sont tenues pour l'ensemble du portefeuille et non pas agence par agence. Les seules statistiques tenues par agence portent sur l'intégralité du portefeuille, tous risques et tous véhicules compris. 
Nous avons donc fait une étude plus précise de l'existant et avons demandé à l'informatique de nous constituer un double fichier d'étude.

(1) un fichier par agence nous indiquant:

- le portefeuille en nombre de véhicules:

- le portefeuille en primes:

4 roues $V_{i}^{1}$

2 roues $V_{i}^{2}$

$P_{i}$

- le nombre de sinistres:

matériels $n_{i}^{m}$

corporels $n_{i}^{c}$

- le montant des sinistres:

matériels $S_{i}^{m}$

corporels $S_{i}^{c}$.

(2) un fichier trié par agence composé de tous les sinistres dépassant $10000 \mathrm{~F}$ fournissant leur nature (matériel ou corporel) et leur montant.

Le travail ayant été effectué en 1979 a porté sur l'exercice 1976 qui, à défaut d'être totalement clos, reflétait déjà des résultats quasiment définitifs.

Afin de fixer les idées, nous publions ci-dessous les premiers chiffres, avant toute vérification, qui sont sortis du listage de ces fichiers:

- nombre d'agences traitées: 1548

- nombre de véhicules:

$$
V=\sum v_{i}=1051188
$$

dont

$$
V^{2}=316567 \quad(2 \text { roues })
$$

- primes totales:

$$
P=799,3 \text { mns F } \quad(\text { mns } F=\text { millions de francs })
$$

- nombre de sinistres:

$$
n=289625
$$

dont

$$
n^{c}=16846 \quad \text { (corporels) }
$$

- montant des sinistres:

$$
S=603,1 \mathrm{mns} \text { F }
$$

dont

$$
S^{c}=336,5 \text { mns F } \quad \text { (corporels) }
$$

- écrêtements:

$$
\begin{aligned}
\text { à } 10000 \mathrm{~F}: & 249,8 \mathrm{mns} F \\
20000 \mathrm{~F}: & 212,1 \mathrm{mns} \mathrm{F} \\
30000 \mathrm{~F}: & 188,2 \mathrm{mns} \mathrm{F} \\
40000 \mathrm{~F}: & 170,5 \mathrm{mns} \mathrm{F} \\
50000 \mathrm{~F}: & 156,0 \mathrm{mns} \mathrm{F}
\end{aligned}
$$

Sinistre maximal: 4,4 mns F. 


\section{B. Raffinage des Données}

Cette étape du travail, qui est la plus ingrate, ne peut être passée sous silence lorsqu'il s'agit de décrire un cas réel.

Un premier examen a fait de suite apparaître trois séries de difficultés:

- De mauvaises codifications faisaient ressortir certains sinistres dans des agences-fantômes qui n'avaient jamais existé, ceci prouvant d'ailleurs qu'il devait exister des erreurs d'imputation, phénomène moins visible.

- Les écrêtements choisis n'étaient pas suffisamment élevés puisque, à $10000 \mathrm{~F}$, on écrêtait $75 \%$ des sinistres corporels et à 50000 F $45 \%$.

- Enfin, on s'est aperçu que le nombre de sinistres matériels dépassant les divers points d'excess n'était pas négligeable et qu'il convenait de les rapatrier en "pseudo-corporels" dans les statistiques en nombre de sinistres du premier fichier.

Afin de rendre les données exploitables, nous avons donc procédé à un travail préparatoire effectué sur un terminal individuel avec des programmes souples, ce qui était possible grâce à la petite taille du fichier décrit ci-dessus.

Cet ensemble de tâches préparatoires au calcul proprement dit s'est enchaîné selon le plan suivant:

1. lecture du fichier d'agences, vérification de séquence et éclatement par compagnies (notre statistique auto portant également sur certaines filiales).

2. préparation du fichier de calcul comportant tous les renseignements documentés et laissant la place pour recevoir les zones d'écrêtement.

3. lecture du fichier de sinistres, vérification de séquence et éclatement par compagnie.

4. totalisation par agence des sinistres dépassant les 5 seuils d'écrêtement retenus $(20000,40000,60000,80000,100000)$ et comptage, en nombre et en montant, des "gros matériels" (dépassant $10000 \mathrm{~F}$ ).

5. assortiment des deux fichiers-rectification des codes erronés (agencesfantômes)-copie des totaux écrêtés dans les zones prévues-rapatriement en nombre et montant des "gros matériels" en "pseudo-corporel".

6. tri par chiffre d'affaires-répartition en 7 groupes d'agences par tranche de 240 (la 7 ème étant incomplète) afin de tester les meilleurs ajustements par niveau de taille.

Ces étapes ont dû être répétées 3 ou 4 fois avant d'obtenir une totale fiabilité.

Le tableau ci-dessous résume la situation avant et après raffinage:

\begin{tabular}{lcc}
\hline \hline & $\begin{array}{c}\text { avant raffinage } \\
\text { voir ci-dessus }\end{array}$ & après raffinage \\
\hline Nombre d'agences & 1548 & 1554 \\
Nombre de véhicules & 1051188 & 1057018 \\
Nombre de 2 roues & 316567 & 318403 \\
Primes totales en mns F & 799,3 & 803,7 \\
Nombre de sinistres & 289625 & 290706 \\
Montant des sinistres & 603,1 & 606,5 \\
Nombre de corporels & 16846 & $18532^{*}$ \\
Montant des corporels & 336,5 & $363,9^{*}$ \\
\hline
\end{tabular}

* Dont 1601 gros matériels pour 26,2 mns $F$ rapatriés en corporels. 
- surcrêtes:

$$
\begin{aligned}
20000 \mathrm{~F}: & 206,7 \mathrm{mns} F \\
40000 \mathrm{~F}: & 164,7 \mathrm{mns} \mathrm{F} \\
60000 \mathrm{~F}: & 138,0 \mathrm{mns} \mathrm{F} \\
80000 \mathrm{~F}: & 122,0 \mathrm{mns} \mathrm{F} \\
100000 \mathrm{~F}: & 107,6 \mathrm{mns} \mathrm{F}
\end{aligned}
$$

\section{Le Module de Calcul}

Ce module avait pour mission de tester quelle était, pour les 5 priorités fixes ci-contre définies, et dans chacun des 7 groupes de taille, la meilleure des méthodes envisagées à savoir:

1. la répartition sur les primes

2. la répartition sur les surcrêtes

3. la répartition sur le nombre de véhicules, et

0 . aucune répartition: sinistres nets sur primes amputées de la prime d'excess.

Pour chaque agence on a donc calculé, à côté du brut, le $S / P$ théorique $\hat{S}_{i} / P_{i}$ défini au chapitre II, à l'aide de la moyenne nationale $m$ du coût moyen corporel

$$
m=\frac{363,9 \mathrm{mns} \mathrm{F}}{18532}=19636 \text {. }
$$

Puis au cours de 4 calculs successifs (type $1,2,3,0$ ) on a calculé, pour chacun des 7 groupes, $h$ le biais moyen

$$
\beta_{t, r}^{h}=\frac{1}{\sum_{i \in h} 1} \times \sqrt{\sum_{i \in h}\left(J_{i}-J_{i}^{\prime}\right)^{2}}
$$

en posant $J_{i}=\hat{S}_{i} / P_{i}$ et $J_{i}^{\prime}$ comme le ratio précédemment exposé selon le type choisi- $\left(S_{i}^{\prime} / P_{i}\right.$ ou $T_{i} / P_{i}^{\prime}$ ayant un mode de calcul différent selon le type $t$ et le seuil d'écrêtement $r$, voir $\S \mathrm{I})$.

Pour être complet, nous ajouterons qu'en ce qui concerne le type 3 (répartition par véhicule), nous n'avons compté les 2 roues que pour une fraction de véhicules, selon une équivalence de prime qui s'est avérée être un 2 roues $=0,126$ véhicule 4 roues.

C'est donc la minimisation du biais de $\beta_{t, r}^{h}$ qui donne la traduction concrète de "meilleur type de calcul" et "meilleur niveau d'écrêtement", expressions qui, à défaut d'être définies, n’avaient pas jusque là de signification réelle.

\section{Les Résultats Obtenus}

Une première étude sur les 240 premières agences sur les types 1,2 et 3 , a permis d'éliminer de suite le dernier comme le fait ressortir le tableau ci-dessous (si bien que l'on n'a pas examiné les autres groupes).

Entre les deux premiers types qui semblaient à très peu de chose près équivalents, nous avons bien sûr choisi le premier pour les raisons politiques exposées ci-dessus. 
Groupe No 1. BIAIS EN \%

\begin{tabular}{rccc}
\hline Ecrêtement & Type No 1 & Type No 2 & Type No 3 \\
\hline 0 & 38,16 & 38,16 & 38,16 \\
20000 & 12,48 & 13,59 & 35,96 \\
40000 & 11,88 & 12,70 & 36,61 \\
60000 & 11,61 & 12,42 & 30,60 \\
80000 & 11,77 & 12,76 & 26,76 \\
100000 & 11,98 & 13,08 & 23,74 \\
\hline
\end{tabular}

L'étude de ce type 1 dans les différents groupes d'agence a fait ressortir les biais suivants:

BIAIS EN \%. PRIMES EN mns F

\begin{tabular}{crccccrr}
\hline \hline Groupe & 1 & 2 & 3 & 4 & 5 & 6 & 7 \\
\hline Par moyenne & 127,02 & 70,07 & 51,42 & 39.04 & 27,59 & 17,16 & 5,37 \\
Sans écrêtement & 38,16 & 34,29 & 36,56 & 53,44 & 65,19 & 52,11 & 215,12 \\
20000 & 12,48 & 13,08 & 13,23 & 14,70 & 17,41 & 18,85 & 23,55 \\
40000 & 11,88 & 11,52 & 12,13 & 13,53 & 15,51 & 16,67 & 27,92 \\
60000 & 11,61 & 10,93 & 11,99 & 13,70 & 15,43 & 16,63 & 37,46 \\
80000 & 11,77 & 11,20 & 12,84 & 14,69 & 16,41 & 18,03 & 48,98 \\
100000 & 11,98 & 11,55 & 13,78 & 15,77 & 17,64 & 19,64 & 59,61 \\
\hline
\end{tabular}

Il est de suite apparu que le groupe 7 des petites agences n'était pas ajustable mais que, ce cas mis à part, on obtenait l'optimum dans une tranche d'écrêtement comprise entre 40 et $60000 \mathrm{~F}$.

On a donc refait le calcul de type 0 (sous-crêtes rapportées aux primes nettes de l'excess) sur une "bande élargie" allant de 45000 à 65000 par pas de 5000 . On a obtenu le résultat ci-après:

\begin{tabular}{crrrrrrr}
\hline \hline Groupe & 1 & \multicolumn{1}{c}{2} & \multicolumn{1}{c}{3} & 4 & \multicolumn{1}{c}{5} & \multicolumn{1}{c}{6} & 7 \\
\hline Par moyenne & 127,02 & 70,07 & 51,42 & 39,04 & 27,59 & 17,16 & 5,37 \\
Sans écrêtement & 38,16 & 34,29 & 36,59 & 53,44 & 55,19 & 52,11 & 215,12 \\
45000 & 11,47 & 8,30 & 8,52 & 9,70 & 11,69 & 11,58 & 49,90 \\
50000 & 11,38 & 8,28 & 8,74 & 10,16 & 12,06 & 12,21 & 50,58 \\
55000 & 11,33 & 8,31 & 9,07 & 10,61 & 12,46 & 12,84 & 51,89 \\
60000 & 11,30 & 8,41 & 9,44 & 11,08 & 12,94 & 13,57 & 53,57 \\
65000 & 11,30 & 8,55 & 9,87 & 11,53 & 13,47 & 14,30 & 55,38 \\
\hline
\end{tabular}

On était ainsi arrivé à cette conclusion fort safisfaisante que le meilleur ajustement était obtenu en utilisant le processus le plus facile à faire adopter: prendre sur les primes un "impôt" répartissant la charge des gros sinistres et tirer le ratio d'après les sous-crêtes. 


\section{CONCLUSION}

Cette étude a permis de prendre une décision. La priorité de $60000 \mathrm{~F}$ pour 1976 a été choisie et on l'a indexée pour les exercices suivants. Aujourd'hui, le calcul est toujours en vigueur.

Lors de l'exécution de ce travail, il n'avait été effectué sur ce sujet que peu d'écrits publiés sauf, à notre connaissance, la thèse de Mlle Hess patronnée par le Groupement Technique Accidents et déjà citée [1].

Depuis lors une étude fort complète utilisant la théorie de la crédibilité a traité à fond ce sujet [2] ainsi que d'autres papiers du même auteur [3].

Bien que ce travail n'ait pas été conçu lors de son exécution dans le cadre strict d'une application de la théorie de la crédibilité, il semble clairement que les résultats concrets obtenus en soient une illustration naturelle et qu'il pourra être repris sous cette optique sans que ses conclusions en soient modifiées.

\section{BIBLIOGRAPHIE}

[1] Hess, Marie-Pascale. Analyse des techniques d'écrêtements des sinistres et application à la branche Automobile. Thèse présentée devant la faculté des Sciences et l'Institut de Statistique des Universités de Paris et devant l'Institut des Actuaires Français.

[2] Gisler, A. (1980). Optimum Trimming of Data in the Credibility Model. Bulletin des Actuaires Suisses 313-325.

[3] Bühlmann, H., Gisler, A., et Jewell, W. (1982). Excess Claims and Data Trimming in the Context of Credibility Rating Procedures. Bulletin des Actuaires Suisses 117-147. 